\title{
醏
}

\section{ANÁLISIS DE LA TEORÍA DE LOS CONTRATOS COMPLETOS E INCOMPLETOS}

El Premio Nobel en Economía 2016 ha sido concedido a Holmström y Hart por sus aportaciones en la teoría de los contratos. Sus aportaciones intentan modelizar los comportamientos y los posibles efectos de relaciones contractuales tan complejas como la gestión pública versus privada de la prestación de servicios públicos, el reparto del excedente entre el gerente y los accionistas de una empresa, y los procesos de integración industrial en función del reparto de los derechos de propiedad.

Palabras clave: teoría de los contratos, principal-agente, derechos de propiedad.

Clasificación JEL: D23, D52, D86.

\section{Introducción}

La Real Academia de las Ciencias de Suecia ha concedido el Premio Nobel en Economía 2016 a Bengt Holmström y Oliver Hart, por sus importantes aportaciones en el campo de la «teoría de los contratos». La economía funciona a través de los contratos, los estudios de los recién galardonados se centran en analizar cómo se puede mejorar la eficiencia de los contratos, teniendo en cuenta los agentes económicos involucrados en ellos, y cómo se beneficia cada una de las partes implicadas en estas operaciones contractuales.

El profesor Bengt Holmström nació en Helsinki en el año 1949. Es profesor de Economía y Empresa en el Massachussets Institute

* Universidad de Castilla-La Mancha.

Versión de diciembre de 2016. of Technology (MIT). En la Universidad de Helsinki realizó los estudios de Matemáticas, Física, Física Teórica y Estadística en 1972 y realizó su doctorado en la Universidad de Starford en el año 1978.

Inicialmente, comenzó a trabajar en el sector privado, en el grupo A. Ahlstrom (19721974). Desde 1978, ha sido profesor e investigador en distintas universidades: Escuela de Economía de Hanken, Universidad de Northwestern, Universidad de Yale, y desde 1994 hasta la actualidad es profesor del MIT. Además, es miembro de la Academia Americana de las Artes y las Ciencias y de las principales academias científicas de Suecia y Finlandia.

El profesor Oliver Hart nació en 1948 en Londres y se graduó en Matemáticas en la Universidad de Cambridge. Posteriormente, obtuvo el doctorado en Economía por la $\triangleright$ 
Universidad de Princeton. Ha sido docente en diversas universidades como las Universidades de Essex y Cambridge, la Universidad de Pensilvania, la London School of Economics y el Massachussets Institute of Technology. Desde 1993, es profesor de la Universidad de Harvard y, desde 1997, está en la cátedra Andrew E. Furer de dicha universidad.

Las aportaciones de Hart y Holmström nos permiten comprender el funcionamiento de los contratos e instituciones de la vida real y, además, exponen las posibles trampas que pueden existir en el diseño de dichos contratos. Existen multitud de contratos tales como los accionistas y la alta dirección, una compañía de seguros y los propietarios de automóviles, o una autoridad pública y sus proveedores.

Teniendo en cuenta que estas relaciones económicas conllevan en ocasiones interés contrapuesto, es fácil la aparición de conflicto entre las partes. La teoría de los contratos pretende ayudar a diseñar las relaciones contractuales de manera que las partes implicadas en dichos contratos tomen decisiones mutuamente beneficiosas.

Un ejemplo clásico, donde se puede observar este problema, surge al separar la propiedad de las empresas (accionista) y la gestión de las misma (gerente). Este hecho crea el problema del riesgo moral, al poder tomar el gerente de la empresa decisiones en su propio beneficio, pero que pueden perjudicar los intereses de los accionistas. Esto puede ser resuelto si el sueldo del gerente está ligado a los resultados de la empresa. Este sistema no está exento de críticas, como que el gerente sea premiado por el simple hecho de haber tenido suerte y no porque haya realizado una adecuada gestión de la empresa.

Por tanto, teniendo en cuenta lo complejo que pueden ser en muchas ocasiones las relaciones económicas recogidas en un contrato, la teoría de los contratos pretende explicar por qué los contratos tienen diversas formas y diseños. Asimismo, otro objetivo de esta teoría es ayudar a la elaboración de los mejores contratos, lo que provocará que las instituciones en la sociedad también funcionen mejor. De forma que estas teorías pretenden responder a preguntas tales como: ¿los proveedores de servicios públicos, como escuelas, hospitales o prisiones, deberían ser propiedad pública o privada? ¿Deberían pagarse salarios fijos a los maestros, trabajadores de la salud y guardias de la prisión o que su remuneración salarial dependiera de su rendimiento? ¿Hasta qué punto los gerentes deben ser pagados a través de programas de incentivos?

La teoría de los contratos no proporciona necesariamente respuestas definitivas o únicas a estas preguntas, el mejor contrato dependerá de la situación específica y el contexto. Sin embargo, el poder de la teoría es que permite identificar con claridad los elementos esenciales a la hora de realizar los distintos contratos (Royal Swedish Academy, 2016).

El objetivo de este artículo es analizar y resumir las principales aportaciones de los recién laureados. Para ello, después de esta breve introducción, en el apartado segundo, se analiza la teoría de los contratos completos. Seguidamente, se analizan en el apartado tercero las extensiones del modelo básico de Holmström. Por su parte, el apartado cuarto se dedica a la teoría de los contratos incompletos de Hart y en el apartado quinto se exponen las principales aplicaciones de los contratos incompletos: las privatizaciones y las finanzas corporativas. Por último, se cierra con un breve apartado de conclusiones. 


\section{Contrato completo: relación entre el principal y el agente}

En este apartado se analizan las contribuciones de Bengt Holmström en la teoría de los contratos. Los problemas de proporcionar incentivos a los empleados han sido considerados desde hace tiempo, pero es en los años setenta cuando se intenta, por parte de los investigadores, encontrar una solución a cómo diseñar los mejores contratos posibles (Holmström, 1979; Shavell, 1979).

En primer lugar, se expondrán los aspectos del modelo básico de Holmström (1979) «contrato óptimo por incentivos», para seguidamente analizar algunos aspectos de las extensiones y desarrollos posteriores de este modelo básico.

En el modelo básico, que parte del modelo Mirrlees (Mirrlees, 1975), se considera que un agente toma una acción a para el intervalo $[\underline{a}, \bar{a}]$, esto genera un coste $c(a)$ para el agente y un beneficio $\beta=b(a)+\varepsilon$ para el principal, donde $\varepsilon$ es el ruido aleatorio. Para analizar los conflictos de interés, asumimos que tanto $b$ como $c$ son funciones crecientes de $a$, de modo que, si todo se mantiene constante, el principal prefiere una elevada a mientras que el agente prefiere una a más baja, por tanto, se puede interpretar a como el esfuerzo del agente. Se asume que $b$ y $c$ son diferenciables, $b$ es cóncava y $c$ es convexa.

Para ver cómo este modelo simple puede explicar diversas relaciones económicas, se supone, por ejemplo, que el agente puede ser un trabajador, un gerente de una empresa, un empresario, un abogado, o un proveedor de servicios públicos. El principal podría ser un empleador, un consejo de administración, un capitalista de riesgo, un cliente, un regulador o una autoridad pública. En muchos de estos contextos, el resultado de la actividad económica es aleatoria, y compartir el riesgo es un aspecto crucial del problema de la contratación.

Asimismo, $t$ denota un pago, o una transferencia, del principal al agente, donde si $t>0$ indica un pago de $P$ a $A$, mientras que si $t<0$ el pago sería al revés. Suponemos que $P$ y $A$ tienen suficientes recursos y, por tanto, en este modelo básico no se consideran las limitaciones financieras. También, asumimos que el principal dispone de más recursos que el agente, y que $P$ es neutro al riesgo y $A$ es adverso al riesgo.

La ecuación [1] recoge la utilidad esperada del principal:

$$
U_{p}=b(a)-E(t)
$$

Y la ecuación 2 recoge la utilidad esperada del agente:

$$
U_{A}=-c(a)+E(t)-\frac{1}{2} r \operatorname{Var}(t)
$$

donde $r>0$ mide el grado de aversión al riesgo.

Si tomamos la suma de las dos utilidades como el mejor punto de referencia. El superávit en este caso sería:

$$
U_{p}+U_{A}=b(a)-c(a)-\frac{1}{2} r \operatorname{Var}(t)
$$

donde el último término es el coste de utilidad de asumir riesgo por parte de $A$. Se supone que la única acción $a^{*} \in[\underline{a}, \bar{a}]$ maximiza $b(a)$ - $c$ (a). Solo se garantiza que $a^{\star}$ es única si $b$ es estrictamente cóncava, $c$ es estrictamente convexa, o ambos. Usando primas para denotar las primeras derivadas, suponiendo que $b^{\prime}(a)>0$ y $c^{\prime}(\underline{a})=0$ garantiza que $a^{*}>\underline{a}$. También, es conveniente suponer que $c^{\prime}(a)$ es muy grande, entonces $a^{*}<\bar{a}$. El superávit total es máximo cuando $a=a^{*}$ y $A$ no tiene riesgo $\operatorname{Var}(t)=0$. Como ya se ha dicho este sería el mejor resultado posible. 
Si la acción a es observable, el principal puede establecer un sistema de incentivos y establecer un contrato directamente vinculado a la acción del agente $t=t(a)$. El principal podría simplemente incrementar la diferencia $t\left(a^{*}\right)-t(a)$ hasta $-c(a)+t\left(a^{*}\right)>-c(a)+t(a)$ para todo $a \neq a^{*}$. El agente podría estar inducido a tomar la acción $a^{*}$ y el principal asumiría todo el riesgo asociado a la variable aleatoria $\varepsilon$. Esto sería eficiente ya que $P$ es neutral al riesgo y $A$ es adverso al riesgo. Con el sistema de pago de incentivos se puede alcanzar una distribución deseable entre el principal y el agente. Para evitar que el agente tome acciones «equivocadas» $a \neq a^{*}$, en los contratos pueden existir elevadas multas pecuniarias $t(a)<0$. El supuesto de realizar una transferencia $t$ condicionada directamente a la acción a puede conducir al clásico problema de riesgo moral.

Posteriormente, se analiza el caso en el que la acción a no es observable directamente o es difícil de medir. En estos casos, surge el problema de riesgo moral, y que el pago se realiza en función de una medida imprecisa. Concretamente, el beneficio del principal se deriva del comportamiento del agente $t=t(\beta)$, donde el beneficio $\beta=b(\mathrm{a})+\varepsilon$, pero es un indicador imperfecto porque depende las fluctuaciones de $\varepsilon$.

Este caso, por ejemplo, se corresponde cuando los accionistas no pueden observar cómo el gerente dirige la empresa, pero sí puede observar el precio de las acciones en bolsa y/o las distintas medidas contables (Murphy, 1985).

Una clave los planteamientos de Holmström (1979) es el informativeness principle, intuitivamente este concepto hace referencia a que la compensación del agente debe depender de variables (señales) que proporcionan información sobre la acción de éste. Formalmente, $P$ se considera para realizar la remuneración $t$ en función de alguna señal $s$ además de $\beta$.

Este resultado tiene importantes implicaciones prácticas. En términos del modelo antes planteado, una señal $s$ que esté correlacionada con el ruido $\varepsilon$ es potencialmente valiosa para el principal, mientras que una señal que no esté correlacionada con $\varepsilon$ sería inútil. En el contexto de la remuneración de la gerencia, el sueldo del encargado debe depender no sólo de las medidas contables y del precio de las acciones propias, sino también de las señales que están correlacionadas con los costes observables, las condiciones de la demanda y los precios de las acciones de las empresas del mismo sector.

Por otro lado, una crítica a este sistema de remuneración es «el pago por suerte», al pagar al gerente por factores exógenos puede ser que obtenga buenos resultados, pero que no sean debidos a su buena gestión (Bertrand y Mullainathan, 2001).

Por su parte, Holmström y Milgrom (1987), consideran demasiado simple el modelo Mirrlees, que una simple acción o hecho económico sirva de medida para la remuneración, incluso esto podría ser negativo ya que al no tener en cuenta el esfuerzo realizado de modo lineal, podría volverse ineficaz para motivar al agente, como lo demuestran algunos trabajos empíricos (Chevalier y Ellison, 1997). Para solucionar este problema, se propone que la remuneración dependa de la productividad, o que el agente comparta el riesgo con el principal.

\section{Extensiones del modelo de contratos completos de Holmström}

Este modelo básico fue completado por Holmström y sus colaboradores en varias direcciones, tales como: el modelo de multitareas; $\triangleright$ 
los incentivos a equipos de trabajo o la promoción profesional a lo largo de la vida laboral.

\subsection{Modelo de multitarea}

En el modelo clásico de riesgo moral, la acción del agente es unidimensional, e interpretada como un «esfuerzo». Sin embargo, en la realidad las acciones o tareas son complejas y multidimensionales, involucrando varias actividades que sólo pueden ser imperfectamente observadas y medidas. Además, recompensar sólo las actividades medibles puede conducir a un comportamiento disfuncional, debido a que los agentes tenderían a concentrarán demasiada atención en las actividades por las que puede ser recompensado (Kerr, 1975).

Por su parte Baker, Gibbons y Murphy (1994) afirman que «la historia de los negocios está llena de empresas que obtuvieron lo que pagaron». Por ejemplo, si la remuneración de un administrador está fuertemente ligado a ganancias a corto plazo, este podría sacrificar inversiones a largo plazo, lo que tendría efectos negativos en los beneficios a largo plazo de la empresa.

Holmström y Milgrom (1991) modelizan estos argumentos teóricos en un modelo de multitarea. Así pues, se supone que un agente toma dos acciones no observadas, $a_{1}$ y $a_{2}$, con una función de coste $c\left(a_{1}, a_{2}\right)$. Se supone que $\delta^{2} c / \delta a_{1} \delta a_{2}>0$ lo que implica que las dos tareas son sustitutivas. La acción $a_{1}$ genera un output medido por $\beta_{i}=b_{i}\left(a_{i}\right)+\varepsilon_{i}$. Teniendo en cuenta la retribución por rendimiento lineal (Holmström y Milgrom, 1987), la transferencia de $P$ a $A$ sería $t\left(\beta_{1}, \beta_{2}\right)=f+k_{1} \beta_{1}+k_{2} \beta_{2}$.

Supongamos que es difícil medir y recompensar la acción $a_{1}$ (la varianza de $\varepsilon_{1}$ es grande), pero fácil de medir y recompensar la acción $a_{2}$ (la varianza de $\varepsilon_{2}$ es pequeña). Dado que $A$ es adverso al riesgo y $P$ es neutral al riesgo, la distribución óptima del riesgo sugiere que la primera tarea debe ser débilmente incentivada ( $K_{1}$ debe ser pequeño). Pero si $a_{1}$ es importante para el principal, $a_{2}$ también debería estar débilmente incentivada (entonces $k_{2}$ debe ser pequeño también) para evitar que el agente concentre todas sus actividad en $a_{2}$.

$S i$ consideramos que $A$ es un maestro de escuela, donde $a_{1}$ podría representar que el maestro fuera capaz de estimular la curiosidad, la responsabilidad, y la capacidad de pensar independientemente, mientras que $a_{2}$ sería los resultados de los exámenes. El conjunto de habilidades recogidas en $a_{1}$ se podrían medir, pero presentaría un ruido considerable y, por tanto, la vinculación del salario del profesor por medio de incentivos supondría correr demasiado riesgo en el cálculo de la remuneración de los maestros.

Por su parte incentivar sólo $a_{2}$, por ejemplo, vinculando el salario del profesor a las calificaciones de los estudiantes en las pruebas estandarizadas, hará que el maestro deje de enseñar el conjunto más amplio de habilidades antes señalado. Por lo tanto, un contrato óptimo para el maestro puede especificar un salario fijo sin pago de incentivo (explícito). Este ejemplo ilustra cómo en situaciones complejas donde se quiere que exista un equilibrio entre las distintas actividades que implica dicho puesto, puede ser más útil no establecer ninguna variable como óptima para medir el «esfuerzo» del agente y establecer salarios fijos.

En definitiva, el modelo multitarea predice que cuando una tarea importante es difícil de evaluar, entonces los incentivos deben ser débiles para todas las demás tareas.

Este modelo multitarea es completado por Holmström y Milgrom (1994) introduciendo $\triangleright$ 
aspectos como los incentivos de alto rendimiento, la propiedad de los trabajadores y la libertad de los trabajadores para organizar su propio trabajo. Todos ellos son instrumentos complementarios para motivar a los trabajadores en los modelos multitarea.

\subsection{Remuneración a los equipos de trabajo}

Muchos procesos de producción requieren la cooperación de muchos agentes. Si sólo es posible medir la producción agregada, esto hace que sea difícil diseñar contratos óptimos para cada agente y, además, puede surgir el problema de que alguno de los agentes implicados engañe sobre el esfuerzo que realiza y, en realidad, se aproveche del trabajo de los demás, ya que la medida de output es conjunta del esfuerzo del equipo.

Este tema fue tratado por Holmström (1982) y se conoce como el problema de riesgo moral en los equipos de trabajo. Holmström (1982) muestra que, si la recompensa a los agentes implica compartir alguna salida conjunta, esta medida siempre será ineficiente.

Asimismo, introduce el concepto de budget breaker, este concepto hace referencia a cómo el principal puede crear incentivos mediante la eliminación de la producción del equipo en caso de rendimiento inferior y externalizar los trabajos que se realizan de modo ineficiente por los equipos.

Igualmene, Holmström (1982) señala el potencial beneficio en términos de los contratos por incentivos formales para equipos, debido a que puede ser útil para eliminar el ruido en el contrato de un agente individual, es decir, el output agregado puede ser una señal útil del esfuerzo de un agente más allá del desempeño individual del agente, y debería entonces ser incluido en el contrato óptimo. Como en el modelo básico, decimos que no se puede saber si los buenos resultados obtenidos por un agente son fruto su esfuerzo individual o de la suerte. Aquí lo que se dice es cómo todos los agentes se ven afectados por los mismos factores externos, si un individuo se esfuerza dentro de un grupo de trabajo le lleva a obtener un elevado output a la empresa. Entonces, según este autor, el resultado del esfuerzo realizado por un individuo dentro de un grupo de trabajo puede servir como señal individual del trabajo que realiza un agente concreto.

\subsection{Promoción profesional del agente}

El hecho de que los agentes que estén interesados por avanzar o promocionar en sus carreras profesionales puede constituir un incentivo para trabajar muy importante, incluso en el caso de salarios fijos. En este sentido, Fama (1980) sostiene que las preocupaciones por la promoción profesional podrían resolver el problema de riesgo moral, sin necesidad de contratos explícitos basados en el rendimiento.

Por su parte, Holmström (1999) introduce los incentivos que suponen la promoción profesional, mediante la siguiente idea: el principal promete salarios futuros más elevados a los agentes que funcionan bien hoy, pero surge la siguiente pregunta: ¿serían creíbles tales promesas?

En este modelo Holmström (1999) considera que la actuación de un agente hoy depende tanto de su esfuerzo como de su capacidad y ambos no son observables. Por tanto, un buen resultado en el presente hace más probable que la capacidad del agente sea alta, y esto $\triangleright$ 
le hace más atractivo no sólo a su principal actual, sino a otros posibles empleadores en el futuro. La competencia por los servicios del agente hace entonces perfectamente creíble que su salario futuro depende de su rendimiento actual. Por lo tanto, tendrá un incentivo el esforzarse al máximo en el presente, incluso si los contratos no contienen ninguna estrategia explícita de incentivos.

En este modelo, se supone que hay dos períodos, $\tau=1$ y $\tau=2$. La capacidad del agente $\theta$ es la misma en ambos períodos. Además, se supone que inicialmente $\theta$ es desconocida tanto para $P$ como para $A$, y se deducirá de los resultados del primer período. En cada período $\tau$, el agente $A$ elige el esfuerzo $a_{\tau} \in[a, \bar{a}]$ y produce un output $\beta_{\tau}=b\left(\theta+a_{\tau}\right)+\varepsilon_{\tau}$. Si se supone que es imposible escribir contratos explícitos basados en el resultado, el salario $w_{\tau}$ del período debe ser independiente de $\beta_{\tau}$. Sin embargo, la competencia en el mercado de trabajo $w_{2}$ asegura que dependerá de $\beta_{1}$. Específicamente $w_{2}$ será igual a la expectativa racional del sobre $\beta_{2}$, condicionado por $\beta_{1}$ obtenido en $\tau=1$. Puesto que el mercado inferirá una capacidad alta cuando la producción del primer período haya sido alta, $w_{2}$ aumenta en función de $\beta_{1}$. Claramente, $A$ no tiene ninguna razón para trabajar duro en el período 2, cuando su carrera está a punto de terminar. Sin embargo, tiene un incentivo para construir una reputación mediante un alto rendimiento al principio de su vida laboral, convenciendo así al mercado que su capacidad.

Este modelo confirma que la promoción profesional puede aliviar el problema de riesgo moral del modelo básico, aunque no se obtiene tampoco un óptimo social, ya que los agentes pueden trabajar excesivamente duro al principio de sus carreras profesionales, mientras que su esfuerzo descenderá considerablemente cuando su vida laboral este próxima a finalizar.

\section{Teoría de los contratos incompletos de Hart}

La medición imprecisa del rendimiento no es el único obstáculo para la elaboración de contratos eficientes. Con frecuencia, las partes no pueden expresar de manera realista los términos contractuales detallados por adelantado. El problema entonces se convierte en cómo diseñar el mejor contrato posible. Oliver Hart y sus colaboradores en la década de los ochenta tratan de solucionar y explicar este problema, y esto hace que surja la conocida teoría de los contratos incompletos.

La idea principal es que un contrato no puede especificar todo lo que las partes deben hacer en futuras eventualidades. Por ello, debe detallar quién tiene el derecho de decidir qué hacer cuando las partes no pueden estar de acuerdo. Así pues, un individuo con este derecho de decisión tendrá más poder de negociación, y será capaz de obtener un mejor acuerdo una vez que la producción se haya materializado. Asimismo, esto obligará al individuo que tenga más poder a tomar determinadas decisiones de inversión, al tiempo que debilita los incentivos de los individuos que gozan de menos derechos de decisión. En situaciones complejas de contratación, la asignación de los derechos de decisión se convierte así en una alternativa al pago por rendimiento, antes analizado.

Los derechos de decisión y derechos de propiedad son el elemento esencial en los contratos incompletos, debido a que los derechos de decisión o control cuidadosamente asignados pueden sustituir a las recompensas contractualmente especificadas. Dado que un medio importante para asignar los derechos de decisión es a través de la propiedad, la teoría de la contratación incompleta genera una rica $\triangleright$ 
teoría de los derechos de propiedad de Hart (1979).

Formalmente, se asume que es imposible detallar directamente un programa de transferencia $t(\beta)$. En cambio, las transferencias pueden realizarse indirectamente mediante otros tipos de acuerdos contractuales, en concreto, mediante la cesión de derechos de propiedad. Además, en este modelo el riesgo compartido no juega ningún papel $y$, por tanto, el término $\varepsilon$ se elimina del modelo.

Consideramos un caso simple donde un agente produce un output que tiene el valor $\beta=b$ (a) para $P$, pero también puede tener otros usos alternativos. Si el valor de la mejor alternativa viene dado por la función diferenciable donde $v(a)$, donde $0<v(a)<b(a)$ y $0<$ $\mathrm{v}^{\prime}(\mathrm{a})<\mathrm{b}^{\prime}(\mathrm{a})$ para todo $a \in[\underline{a}, \bar{a}]$ ex ante (antes de que $A$ elija $a$ ) las partes deciden quién será el propietario del output final. Si ni a ni $\beta$ se pueden contratar, si $P$ es el propietario y $A$ no obtiene ninguna parte de $\beta$, entonces se ajustará $a=\underline{a}$ para minimizar $c(a)$. Por el contrario, si $A$ tiene la propiedad de $\beta$, puede negar $P$ el output y obtener al menos $v(a)$. Pero un resultado aún mejor se obtendría si $A$ comercia con $P$, después de que la producción haya sido producida. Si las dos partes tienen el mismo poder de negociación, la transferencia resultante será:

$$
t=v(a)+\frac{1}{2}(b(a)-v(a))=\frac{b(a)+v(a)}{2}
$$

Anticipando este resultado, si $A$ es propietario, elige a para maximizar $-c(a)+(b(a)+v(a))$ $/ 2$, en este caso $A$ escoge a mayor que a pero más pequeño que $a^{*}$, así el excedente será mayor si $A$ también es propietario, pero el resultado es menor que el obtenido en el modelo básico inicialmente expuesto.

En relación con este tema, y relacionado con la teoría de la empresa, el también premio nobel Ronald Coase sostiene que las empresas pueden organizar ciertas transacciones con más eficacia que los mercados (Coase, 1937). A diferencia de las transacciones de mercado, la mayor parte de la actividad económica dentro de las empresas no está regulada por contratos explícitos. En este sentido, el laureado Oliver Williamson desarrolló estas ideas más a fondo y creó una rica teoría (basada en contratos incompletos), conocida como economía de costes de transacción (Williamson 1971, 1975, 1979, 1985). Williamson enfatizó inicialmente las ineficiencias ex post creadas por la negociación, pero la atención se dirigió más adelante hacia los incentivos para hacer inversiones específicas de la relación ex ante.

Por su parte, Grossman and Hart (1986) desarrollan la teoría de los costes y beneficios de los procesos de integración de las empresas. En su modelo estudian los incentivos para realizar inversiones no contractuales, que dependen de la propiedad de los activos. En su modelo, consideran dos empresas, una como proveedor «aguas arriba» y un productor «aguas abajo» que han de cooperar para producir un bien final. Ambas partes hacen inversiones específicas. Los contratos son incompletos en el sentido de que ambas empresas no pueden comprometerse ni en los niveles de inversión ni en el reparto del excedente. El incentivo de cada empresa depende de sus expectativas sobre cómo se compartirá el excedente, que a su vez depende de la propiedad de los activos físicos. Las posibles estructuras de propiedad son el proveedor que posee todos los activos (integración vertical ascendente), el productor que posee todos los activos (integración vertical descendente) o cada uno posee sus propios activos (no integración). El modelo incorpora varios elementos claves de la teoría de los costes transacción: contratación incompleta, inversiones específicas y captura del output. Sin embargo, en el modelo $\triangleright$ 
de Grossman y Hart (1986), los costes y beneficios de las diversas estructuras de propiedad se derivan únicamente de su impacto en las inversiones realizadas por cada una de las partes. La teoría no considera las posibles ineficacias ex post causadas por la negociación o la toma de decisiones burocráticas.

Para ver cómo la propiedad de los activos físicos determina los incentivos en contratos incompletos, se parte de nuevo del marco teórico principal-agente. Se supone que $A$ tiene que realizar una inversión en capital humano (conocimiento) que se necesita para que la producción tenga lugar. Para simplificar se considera que $P$ no hace ninguna inversión en este ámbito. Para producir output se necesita capital físico, maquinarla. Si $A$ no tiene acceso a la maquinaria, no puede haber producción. Este aspecto es crucial, puesto que quien posee la maquinaria decide quién tiene acceso a ella, es decir, la propiedad viene con un derecho de veto, y esto influirá en los términos de intercambio. Si $A$ posee la maquinaria, reforzaría su poder en las negociaciones con $P$, aumentando así su parte del excedente. A su vez, esto aumenta el incentivo de $A$ para invertir en capital humano.

Si $P$ posee la maquinaria hay integración vertical y si $A$ posee la maquinaria no hay integración. Si $P$ posee la maquinaria, $P$ tiene el derecho decidir si $A$ puede o no usarla. Asimismo, se supone que $A$ y $P$ dividen el beneficio $b(a)$ en partes iguales, así la transferencia de $P$ a $A$ es $t=b(a) / 2$. Por lo tanto, en este caso, $A$ elige una inversión $a_{p}$ que maximiza $b(a) / 2-c(a)$. Si recordamos en el modelo básico el óptimo era $a^{*}$ que maximiza $b(a)-c(a)$, por tanto, se deduce que hay una inversión insuficiente, ya que $a_{p}<a^{*}$.

Por su parte, si $A$ posee maquinaria, $A$ no dependería de $P$ para poder producir, ya que $A$ puede obtener unilateralmente $v(a)$ del uso alternativo de la maquinaria. Puesto que $b(a)>v(a)$ es previsible que $P$ y $A$ todavía acepten negociar, pero ahora los términos podrían ser más favorables a $A$. Intuitivamente, la parte de $A$ del excedente aumentará. Siguiendo a Grossman y Hart (1986), supongamos que la transferencia de $P$ a $A$ será:

$$
t=v(a)+(b(a)-v(a)) / 2
$$

Es decir, las dos partes dividen la diferencia entre lo que podrían ganar por sí mismo y lo que pueden ganar conjuntamente. Así, si $A$ es propietario de maquinaria, $A$ realiza una inversión $a_{A}$ que maximice:

$$
-c(a)+v(a)+(b(a)-v(a)) / 2
$$

La solución satisface $a_{p}<a_{A}<a^{*}$, es decir, que si $A$ posee maquinaria el excedente es mayor que si la propiedad de la maquinaria es de $P$. Aunque como se puede observar, esta opción sigue siendo menor que la solución óptima que se obtuvo en el modelo básico.

El modelo básico de dos agentes es simétrico y fácil de comprender, pero no permite considerar grandes corporaciones con múltiples divisiones, clientes y proveedores, y tal vez miles de empleados. Posteriormente, Hart y Moore (1990) desarrollan la teoría de los derechos de propiedad para un entorno con muchas partes implicadas, varios activos, lo que proporciona un marco de producción más adecuado.

Inicialmente, se va a mantener el supuesto de que hay sólo dos partes, $A$ y $P$, pero ahora hay muchos activos productivos y ambas partes invierten en capital humano. Así pues, sean a y $p$ los niveles de inversión de $A$ y $P$, respectivamente. Sea $c_{\mathrm{A}}(a)$ la función de coste de $A$, y la $c_{p}(p)$ función de coste de $P$; $v(i)$ representa la opción de no cooperar. Por último $\beta$ es el beneficio que obtiene $P$ de cooperar con $A$.

Si $\beta>v_{A}+v_{\mathrm{P}}$ entonces la cooperación es eficiente, puesto que se ha asumido teóricamente que no existen ineficiencias ex post, $D$ 
$y$, por tanto, se espera que ambas partes decidan cooperar. Pero la opción de no cooperar también es importante, ya que influye en el pago o en la transferencia de $P$ a $A$. Además, esas opciones dependen de la asignación de derechos de propiedad, porque si las partes deciden separarse, entonces la parte que posee un activo puede impedir que el otro lo utilice. Por consiguiente, la transferencia de la propiedad de un activo particular de $P$ a $A$ aumentará $v_{\mathrm{A}}$ y reducirá $v_{\mathrm{p}}$ (pero afectará a $\beta$, ya que todos los activos se utilizarán cuando $A$ y $P$ cooperen).

De este modo la transferencia $t$ iguala las ganancias netas de la cooperación: $t-v_{A}=\beta-t-v_{p}$ Despejando $t$, obtiene

$$
t=1 / 2\left(\beta+v_{\mathrm{A}}-v_{p}\right)
$$

$A$ escogerá a para maximizar su rentabilidad

$$
\pi_{A}=t-c_{A}(a)=1 / 2\left(\beta+v_{A}-v_{p}\right)-c_{A}(a)
$$

Mientras que $P$ elegirá $p$ para obtener su máximo beneficio

$$
\pi_{\mathrm{B}}=\beta-t-c_{p}(p)=1 / 2\left(\beta-v_{A}+v_{p}\right)-c_{p}(p)
$$

Si las partes no cooperan, no pueden beneficiarse del capital humano de la otra parte, por lo que $v_{\mathrm{A}}$ es independiente de $p, \mathrm{y} v_{\mathrm{p}}$ es independiente de $a$. Por lo tanto, los incentivos para invertir dependen de las derivadas de:

$$
\begin{gathered}
\frac{\partial \pi_{A}}{\partial a}=\frac{1}{2}\left(\frac{\partial \beta}{\partial a}+\frac{d v_{A}}{d a}\right)-\frac{d c_{A}}{d a} \\
\frac{\partial \pi_{P}}{\partial p}=\frac{1}{2}\left(\frac{\partial \beta}{\partial p}+\frac{d v_{p}}{d p}\right)-\frac{d c_{p}}{d p}
\end{gathered}
$$

La asignación de los derechos de propiedad, como se observa en las derivadas anteriores, depende de las opciones alternativas a cooperar. En este caso, transferir la propiedad de cualquier activo de $P$ a $A$ eleva $d v_{\mathrm{A}} / d a$ pero reduce $d v_{\mathrm{p}} / d p \mathrm{y}$, por tanto, eleva el incentivo de $A$ para invertir, pero reduce el incentivo de $P$ para hacerlo. En el caso especial de dos partes, los resultados de Hart y Moore (1990) indican que si la inversión de una parte se vuelve más importante (en términos de aumentar el beneficio), entonces esta parte debe poseer más activos.

Además, Hart y Moore (1990) concluyen que la propiedad conjunta es ineficiente porque si cada parte puede sostener a la otra parte (negarle el uso del activo después de la separación), los incentivos se debilitan para ambas partes. Por la misma razón, los activos estrictamente complementarios deben ser propiedad conjunta. La propiedad externa siempre es ineficiente, ya que quien no pertenece al acuerdo de cooperación puede negar a ambas partes el uso del activo.

Cuando hay más partes implicadas en un contrato, Hart y Moore (1990) usan el valor de Shapley (Shapley, 1953), lo que les permite predecir una rica variedad de estructuras de propiedad, dependiente de quiénes sean los actores clave, además, en este tipo de modelos las decisiones deben ser tomadas por el voto de la mayoría, en función de los derechos de propiedad que tengan los agentes implicados.

La teoría de los contratos incompletos ha sido criticada por hacer suposiciones aparentemente fuertes y en ocasiones arbitrarias, (Noldeke y Schmidt, 1994; Tirole, 1999; Maskin y Tirole, 1999). Estas críticas sostienen que la teoría de los contratos incompletos era que la información sobre tales variables que se suponen que son difíciles de medir, podrían, al menos en teoría, ser obtenidas de las partes contratantes usando «juegos de mensajes» descritos por Maskin (1977).

\section{Aplicaciones de la teoría de los contratos incompletos}

La teoría de los contratos ha tenido diversas aplicaciones en ámbitos tales como: el $\triangleright$ 
debate sobre la propiedad pública versus la propiedad privada y las finanzas cooperativas.

\subsection{Propiedad pública versus propiedad privada}

La teoría de los contratos incompletos ha sido utilizada para comprender los costes y beneficios de las privatizaciones (Hart, 2003). En un modelo de contratación completo, la propiedad pública o privada no importa, ya que la relación entre el Gobierno y la empresa puede ser totalmente resuelto en un contrato de incentivos detallado. Por el contrario, cuando los contratos son incompletos, la identidad del propietario se vuelve muy relevante, porque el propietario conserva los derechos residuales de control.

En este caso, el modelo se especificaría del siguiente modo: $P$ es el Gobierno que desea la producción de algún servicio público, y $A$ es el gerente encargado de producir este servicio, igual que en los casos anteriores, la propiedad de los activos determina el poder de negociación y los incentivos para actuar. En el contexto de la privatización, el Gobierno se preocupa por la eficiencia de la producción y la calidad del servicio. Pero la calidad de los servicios sociales es difícil de especificar en un contrato, mientras que el contratista privado tiene como incentivo producir al menor coste posible.

Por su parte Hart, Shleifer y Vishny (1997) analizan las privatizaciones a partir del enfoque de contratos incompletos y el modelo multitarea de Holmström y Milgrom (1991). En este trabajo se supone que una innovación reduce los costes y beneficios del uso de un activo. El propietario del activo tiene el derecho de implementar la innovación o evitar su implementación.

En concreto, la innovación reduce el beneficio de $P$ de $b_{0}$ a $b_{0}-z(a)$ y el coste de $A c_{0}$ a $c_{0}-m(a)$. Además, $z$ (a) y $m(a)$ aumentan en a, y $a \in[\underline{a}, \bar{a}]$ se interpreta como una actividad que ahorra de costes, pero que reduce la calidad del servicio que recibe del agente. Asimismo, se considera que la innovación es eficiente, pues su implementación eleva el superávit total en $m(a)-z(a)>0$ para todo $a \in$ [a, $\bar{a}$ ]. El óptimo $a^{*} \in(0, \bar{a})$ que cumple que $m^{\prime}\left(a^{*}\right)=z^{\prime}\left(a^{*}\right)$ es decir, la ganancia del ahorro de costes se debe compensar con la pérdida de la reducción de la calidad.

$S i$ es propietario de la innovación, no necesita aprobación de $P$ para implementar la innovación de ahorro de costes, para reducir los costes tanto como sea posible $-c_{0}+m(a)$, es decir, establece $a=\bar{a}$. Este resultado es altamente ineficiente puesto que $A$ puede unilateralmente implementar la innovación de reducción de costes, y no tener en cuenta la calidad del servicio que presta a $P$.

Si la propiedad es de $P$, el coste de $A$ es $C_{0}$, a menos que obtenga la aprobación de $P$ para implementar la innovación. Si como en el caso anterior, las negociaciones entre $A$ y $P$ conducen a una división igual del excedente, que es igual a $m(a)-z(a)$, puesto que la recompensa de $A$ será $-c_{0}+(m(a)-z(a)) / 2$ y tendrá los incentivos correctos para negociar el intercambio entre costes y calidad del servicios, porque $A$ maximizará su recompensa eligiendo $a^{*}$, tal que $m^{\prime}\left(a^{*}\right)-z^{\prime}\left(a^{*}\right)=0$ Por tanto, cuando $P$ es propietario el resultado es eficiente, debido a que cuando $P$ tiene el derecho de rechazar la innovación y $A$ debe tener en cuenta los intereses de $P$.

Este modelo de Hart, Shleifer y Vishny (1996) también da una visión más general de este tipo de contratos al asumir que el agente puede hacer dos tipos diferentes de innovaciones: innovaciones de costes e innovaciones de calidad. Un proveedor de servicios propiedad del Gobierno $(P)$ tendrá poco incentivo para invertir $D$ 
en la innovación, mientras que un contratista privado tendrá incentivos más fuertes tanto para mejorar la calidad como para reducir los costes, pero demuestran que el contratista privado tiene incentivos más fuertes por reducir los costes que por aumentar la calidad. En general, cuanto mayores sean las consecuencias adversas de la reducción de costes en la calidad, mayor deberá ser la propiedad gubernamental. Este análisis proporciona argumentos en contra de la externalización y privatización de servicios públicos como sanidad, educación y prisiones, debido a la bajada en la calidad del servicio.

\subsection{Finanzas corporativas}

La aplicación más importante de la teoría de los contratos incompletos se ha dado en el ámbito de la empresa, puesto que es cuestión crucial asegurar que los gerentes y administradores actúen a favor de los inversores. En este ámbito, Grossman y Hart (1982) se cuestionan quién debe tener los derechos de control residual, es decir, el derecho a tomar decisiones que no están explícitamente decididas en un contrato ex ante y qué pasa con la asignación de estos derechos de decisión si una firma incumple una deuda.

En este trabajo se plantea el caso de una empresa, en el que es difícil de determinar si la gestión de un gerente es adecuada o no, ya que éste puede desviar los beneficios de la empresa en su propio beneficio. La mejor solución puede ser que el gerente se convierta en empresario y sea dueño de la propia empresa. Un empresario puede decidir libremente cómo dirigir la empresa y hacer el intercambio apropiado entre las acciones que aumentan los beneficios y las acciones que aumentan sus beneficios privados.
La limitación de esta solución es que el gerente a veces no puede permitirse comprar la empresa, por lo que los inversores externos tienen que financiar la compra. Pero si los beneficios no pueden ser conocidos cuando se firma el contrato, ¿cómo pueden los inversores asegurarse de que obtendrán su dinero? Una solución es prometerles un pago fijo futuro con garantías, independientemente de los beneficios. Si el pago no se hace, la propiedad se transfiere a los inversores, que pueden liquidar los activos de la empresa.

En términos más generales, la teoría del contrato incompleto predice que los empresarios deberían tener el derecho de tomar la mayoría de las decisiones en sus empresas siempre y cuando el rendimiento sea bueno, pero los inversionistas deberían tener más derechos de decisión cuando los resultados empresariales se deterioran. Esta característica es típica de los contratos financieros del mundo real, como los sofisticados contratos firmados por los empresarios y los capitalistas.

\section{Conclusiones}

La teoría de los contratos analiza los obstáculos y las posibles soluciones en las relaciones económicas entre los distintos agentes implicados. Los recién premiados Bengt Holmström y Oliver Hart, han intentado modelizar y buscar soluciones a las relaciones contractuales tan complejas como las que se dan entre un gerente y un accionista, el Gobierno y los suministradores de los servicios públicos, y/o la retribución de los trabajadores cuando el rendimiento de estos es difícil de medir. También, han propuesto nuevas formas de diseñar los contratos, tanto en los mercados privados como en el ámbito de las políticas públicas. 
Asimismo, es necesario señalar que cada uno de ellos se ha centrado en variables clave diferentes: Holmström ha tratado de resolver el problema de riesgo moral que surge cuando se intenta remunerar en función del output obtenido y de diseñar contratos que consigan un equilibrio entre los intereses de principal y el agente. En cambio, Hart analiza cómo la distribución de los derechos de propiedad pueden influir en el funcionamiento eficiente de determinadas relaciones económicas como la prestación de servicios públicos, la innovación, o las finanzas corporativas.

\section{Bibliografía}

[1] BAKER, G.; GIBBONS, R. y MURPHY, K. (1994). «Subjective Performance Measures in Optimal Incentive Contracts». The Quarterly Journal of Economics, vol. 109, $\mathrm{n}^{\circ} 4$, pp. 1125-1156.

[2] BERTRAND, M.; y MULLAINATHAN, S. (2001). «Are CEOs Rewarded for Luck? The Ones without Principals Are». Quarterly Journal of Economics, $n^{\circ} 116$, pp. 901-932.

[3] COASE, R.H. (1937). "The Nature of the Firm». Economica, $n^{\circ}$ 4, pp. 386-405.

[4] FAMA, E. (1980). «Agency Problems and the Theory of the Firm». Journal of Political Economy, ${ }^{\circ} 88$, pp. 288-307.

[5] GROSSMAN, S. y HART, O. (1982). "Corporate Financial Structure and Managerial Incentives». In: The Economics of Information and Uncertainty (ed. J. McCall).

[6] GROSSMAN, S. y HART, O. (1986). «The Costs and Benefits of Ownership: A Theory of Vertical and Lateral Integration». Journal of Political Economy, n 94 , pp. 691-719.

[7] HART, O. (1979). «Monopolistic Competition in a Large Economy with Differentiated Commodities". Review of Economic Studies, $n^{\circ} 46$, pp. 1-30.

[8] HART, O. (2003). «Incomplete Contracts and Public Ownership: Remarks, and an Application to Public-Private Partnerships». Economic Journal, $\mathrm{n}^{\circ} 113$, Conference Papers, C69-C76.
[9] HART, O.; SHLEIFER, A. y VISHNY, R. (1997). «The Proper Scope of Government: Theory and an Application to Prisons». Quarterly Journal of Economics, $\mathrm{n}^{\circ} 112$, pp. 1127-1161.

[10] HART, O. y MOORE, J. (1990). «Property Rights and the Nature of the Firm". Journal of Political Economy, no 98, pp. 1119-1158.

[11] HOLMSTRÖM, B. (1979). «Moral Hazard and Observability». Bell Journal of Economics, $n^{\circ}$ 10, pp. 74-91.

[12] HOLMSTRÖM, B. (1982). «Moral Hazard in Teams». Bell Journal of Economics, $n^{\circ} 13$, pp. 324-340.

[14] HOLMSTRÖM, B. (1999). «Managerial Incentive Problems a Dynamic Perspective». Review of Economic Studies, $n^{\circ} 66$, pp. 169-182.

[15] HOLMSTRÖM, B. y MILGROM, P. (1987). "Aggregation and Linearity in the Provision of Intertemporal Incentives». Econometrica, $n^{\circ}$ 55, pp. 303-328.

[16] HOLMSTRÖM, B. y MILGROM, P. (1991). «Multi-Task Principal Agent Analysis». Journal of Law, Economics and Organization, $\mathrm{n}^{\circ} 7$, pp. 24-52.

[17] MASKIN, E. (1977). «Nash Equilibrium and Welfare Optimality». Paper presented at the summer workshop of the Econometric Society in Paris, June 1977, publicado 1999 in the Review of Economic Studies, $n^{\circ} 66, p p$. 23-38.

[18] MASKIN, E. y TIROLE, J. (1999). «Unforeseen Contingencies and Incomplete Contracts». Review of Economic Studies, $\mathrm{n}^{\circ} 66$, pp. 83-114.

[19] KERR, S. (1975). «On the Folly of Rewarding A, while Hoping for B». Academy of Management Journal, $\mathrm{n}^{\circ} 18$, pp. 769-783.

[20] MIRRLEES, J. A. (1975). «The Theory of Moral Hazard and Unobservable Behaviour", Part I, Mimeo, Oxford, reeditado en Review of Economic Studies, $\mathrm{n}^{\circ}$ 66, pp. 3-21.

[21] MURPHY, K. (1985). «Corporate Performance and Managerial Remuneration: An Empirical Analysis». Journal of Accounting and Economics, $\mathrm{n}^{\circ}$ 7, pp. 11-42.

[22] NOLDEKE, G. y SCHMIDT, K. (1994). "Option Contracts and Renegotiation: A Solution to the Hold-up Problem». Rand Journal of Economics, $\mathrm{n}^{\circ}$ 26, pp. 163-172.

[23] ROYAL SWEDISH ACADEMY (2016). «Oliver Hart and Bengt Holmström: Contract Theory». Disponible en: https://www.nobelprize.org/ 
[24] SHAPLEY, L. (1953). «A Value for n-Person Games, in Contributions to the Theory of Games», vol. 2. (eds. H. Kuhn and A. Tucker), Princeton University Press, Princeton NJ.

[25] SHAVELL, S. (1979). «Risk Sharing and Incentives in the Principal and Agent Relationship". The Bell Journal of Economics, vol. $10, \mathrm{n}^{\circ} 1$ pp. 55-73.

[26] TIROLE, J. (1999). «Incomplete Contracts: Where do We Stand?» Econometrica, $\mathrm{n}^{\circ} 67$, pp. 741-781.
[27] WILLIAMSON, O. (1971). «The Vertical Integration of Production: Market Failure Considerations". American Economic Review Papers and Proceedings, $\mathrm{n}^{\circ}$ 61, pp. 112-123.

[28] WILLIAMSON, O. (1975). Markets and Hierarchies. New York: Free Press.

[29] WILLIAMSON, O. (1979). «Transaction-Cost Economics: The Governance of Contractual Relations». Journal of Law and Economics, $\mathrm{n}^{\circ} 22$, pp. 233-271.

[30] WILLIAMSON, O. (1985). The Economic Institutions of Capitalism. New York: Free Press. 